\title{
Vorwort zu Band 3/1
}

Materialien und Materialsysteme bilden das Fundament der Nanotechnologie und sind in ihren unterschiedlichen Ausprägungen Gegenstand der Nanostrukturforschung. Materialien und Systeme können insbesondere gleichsam die Materialisation völlig neuartiger Phänomene sein, wobei hier häufig die materielle Voraussetzung im nanostrukturierten Aufbau der entsprechenden Materialien und Systeme begründet liegt. Diesem Sachverhalt widmet sich in der Hauptsache Band 3 dieser Buchreihe. Wiederum sind viele der vorgestellten Materialien und Systeme quasi funktionelle Manifestationen vieler in Band 1 der Buchreihe behandelter Grundlagen und komplettieren die bereits in Band 2 behandelten Materialien und Systeme. Auch wenn die Bezüge zwischen allen Bänden der vorliegenden Lehrbuchreihe eng sind und Bezüge auch häufig explizit vermerkt werden, so ist ebenfalls Band 3/1 so konzipiert, dass er den enthaltenen Stoff in einer Weise vermittelt, dass ein detailliertes Studium der Bände 1 und 2 nicht zwingend vorausgesetzt wird. Materialien, Systeme und Methoden werden also umfassend erklärend und mit einem optionalen Bezug $\mathrm{zu}$ bereits behandelten Sachverhalten dargestellt. Wiederum werden theoretische, experimentelle und technische Aspekte gleichgewichtig und mit deutlichem Bezug zu allerneuesten Forschungsergebnissen behandelt.

Wie wohl kein anderes Objekt der Nanostrukturforschung und Nanotechnologie werden Nanopartikel mit den Forschungs- und Anwendungsfeldern assoziiert. Dies ist in gewisser Weise sogar berechtigt, weil es a priori eine schier unendliche Vielzahl unterschiedlich zusammengesetzter und geformter Nanopartikel gibt, aber auch, weil zu den spezifischen Eigenschaften dieser Form der Materie und zu ihren Anwendungen in den letzten Jahren ein hoher Forschungs- und Entwicklungsaufwand betrieben wurde. Nanopartikel können heute als isolierte Objekte analysiert und charakterisiert werden, in ihrem Transport durch lebende Organismen verfolgt oder auch im Verbund zu funktionellen neuen Materialien verarbeitet werden. Für die Herstellung von Nanopartikeln wurden neuartige Herstellungsverfahren entwickelt, die teilweise Massenproduktion zulassen und von größter industrieller Bedeutung sind. Thermodynamische und kinetische Aspekte sind bei der Herstellung, aber auch für die Langzeitstabilität der Teilchen äußerst relevant und werden im vorliegenden Band eingehend behandelt, genauso wie die spezifischen mechanischen, elektronischen, optischen oder auch magnetischen Eigenschaften der winzigen Partikel.

Besondere mechanische Eigenschaften von Nanopartikeln sind Grundlage für viele bereits heute bestehende technische Anwendungen neuartiger nanostrukturierter Materialien und Oberflächen. Im Hinblick auf elektronische Eigenschaften können sich bestimmte Nanopartikel wie nulldimensionale Objekte verhalten, und man spricht folgerichtig von Quantenpunkten. Quantenpunkte als Bestandteile elektronischer oder optoelektronischer Bauelemente haben bereits heute ein enormes Anwendungspotential und sind gleichzeitig dennoch Gegenstand einer außerordent- 
lich dynamischen Forschung. Magnetische Nanopartikel wiederum werden seit langem beispielsweise in Speichermedien eingesetzt und sind natürlich wesentlicher Bestandteil der Ferrofluide mit ihren vielfältigen Anwendungen. Ihre Verfügbarkeit führte allerdings in den letzten Jahren auch zu neuartigen pharmakologischen Ansätzen. Die chemischen Eigenschaften von Nanopartikeln sind von Bedeutung etwa im Bereich katalytischer Verfahren oder auch im Hinblick auf die weitere Funktionalisierung der Partikel sowie im Hinblick auf ihr Verhalten in biologischer Umgebung.

In Band 2 hatten wir ausführlich die Kohlenstoffgrundbausteine in Form von Graphen, von Kohlenstoffnanoröhrchen und in Form der Fullerene behandelt. Die entsprechenden Kohlenstoffallotrope etablieren quasi zwei-, ein- und nulldimensionale Systeme. Seit einigen Jahren ist allerdings bekannt, dass die Niedrigdimensionalität nicht auf das Element Kohlenstoff allein beschränkt ist, sondern, dass sich aus zahlreichen weiteren Elementen und insbesondere auch aus Verbindungen ebenfalls niedrigdimensionale Systeme realisieren lassen. Das gilt insbesondere für zweidimensionale graphenartige Systeme. So wichtige Materialien wie Silizium oder Germanium können ebenfalls in Form von Monolagen präpariert werden, genauso wie ÜbergangsmetallDichalcogenide und weitere Graphenanaloga. Selbst bestimmte Oxide und Hydroxide oder auch Polymere können in einer zweidimensionalen Konfiguration vorliegen. Dieser Sachverhalt motiviert im vorliegenden Band eine umfassende Diskussion der physikalischen Eigenschaften niedrigdimensionaler und insbesondere zweidimensionaler Systeme. Diese umfasst elektronische, optische, aber auch thermische Eigenschaften.

Neben zweidimensionalen Systemen lassen sich auch ein- und nulldimensionale Systeme aus weiteren, insbesondere auch aus anorganischen Materialien realisieren, was Begriffe wie Nanoröhrchen, Nanodrähte und Quantenpunkte begründet. Je nach Dimensionalität haben die entsprechenden Systeme Eigenschaften, die völlig neuartige technologische Anwendungen ermöglichen. Derartige Anwendungen reichen von neuartigen Formen des Arzneimitteltransports über Verbundmaterialien mit bisher unbekannten Eigenschaften bis hin zu Einzelphotonenquellen. Die Vielfalt der niedrigdimensionalen Systeme wird im vorliegenden Band umfassend dargestellt. Grundlegende dimensionsabhängige Aspekte werden herausgestellt und Anwendungspotentiale in Form von Realisationen oder auch von absehbaren Möglichkeiten abgeschätzt.

Metamaterialien subsumieren, wie es der Begriff auch impliziert, unterschiedliche Materialien in unterschiedlichen Strukturen, die gemeinsam ein übergeordnetes Material mit effektiven Eigenschaften konstituieren. Dabei sind besonders interessant solche Materialien, in denen definierte Nanostrukturen eines Materials regelmäßig eingebettet sind in ein umgebendes Material. Hierbei kann es sich beispielsweise um metallische Nanopartikel in einer dielektrischen Matrix handeln. Besonders spektakuläre Eigenschaften haben elektromagnetische Metamaterialien, die beispielsweise Materialien mit einem negativen optischen Brechungsindex umfassen. Optische Metamaterialien ermöglichen konzeptionell die Realisierung völlig neuer Bauelemente, 
wie beispielsweise „Superlinsen“ oder „Tarnkappen“. Aber auch magnonische oder mechanische Metamaterialien sind von zunehmender Bedeutung. Im vorliegenden Kontext werden die typischen strukturellen Eigenschaften von Metamaterialien behandelt, und ein Formalismus wird präsentiert, der insbesondere die Beschreibung der photonischen Eigenschaften nanostrukturierter Metamaterialien ermöglicht.

Eine so ausgesprochen umfangreiche und detaillierte Darstellung der Nanostrukturforschung und Nanotechnologie auf der Basis modernster Forschungsergebnisse wäre nicht denkbar, wenn nicht zahlreiche Kolleginnen und Kollegen weltweit ihre Forschungsergebnisse zur Verfügung gestellt hätten. Insbesondere ist die Vielzahl der vorgestellten Ergebnisse aus Grundlagenforschung, angewandter Forschung und Anwendung die Basis dafür, dass das vorliegende Buch wie auch die anderen Bände der Reihe einen ausgesprochen interdisziplinären Charakter besitzt. Ich möchte mich daher bei allen Kolleginnen und Kollegen, die im Zusammenhang mit den entsprechenden Ergebnissen zitiert wurden, explizit für ihre spannenden Ergebnisse bedanken.

Auch im vorliegenden Fall war für die Bearbeitung oder Herstellung der zahlreichen Abbildungen in diesem Buch Frau Gabriele Kreutzer-Jungmann verantwortlich, bei der ich mich für ihre außerordentlich professionelle Arbeit und für ihr großes Maß an Geduld sehr bedanken möchte. Die Lösung der vielen komplexen Formatierungsprobleme und die Erstellung des druckfertigen Manuskripts lag erneut bei Frau Stefanie Neumann, ohne deren umfangreiche Expertise, große Akribie und erhebliche Geduld die Realisierung dieses Buches in der vorliegenden Form nicht möglich gewesen wäre. Dafür bedanke ich mich herzlich. Zur Reduzierung der Anzahl der Fehler des Buches haben eine Reihe von Personen beigetragen. Für einen ganz erheblichen Beitrag möchte ich an dieser Stelle erneut Herrn Harro Hartmann danken.

Von unschätzbarer Bedeutung für mich war die geduldige und sachkundige Begleitung durch den DeGruyter-Verlag, der die gesamte Buchreihe nun schon seit einigen Jahren betreut. Stellvertretend für das gesamte Team möchte ich hier insbesondere die angenehme Kooperation mit Frau Nadja Schedensack und Frau Kristin Berber-Nerlinger nennen. 
